\title{
Global profiles of a cetylated proteins in the brains of scrapie agents 139A- and ME7-infected mice collected at mid-early, mid-late and terminal stage
}

qi Shi

Chinese Centers for Disease Control and Prevention

Dong-Dong Chen

Chinese Center for Disease Control and Prevention

Maimaitiming Adalaiti

Chinese center for disease control and prevention

Kang Xiao

Chinese center for disease control and prevention

Li-Ping Gao

Chinese center for disease control and prevention

Xue-Hua Yang

Chinese center for disease control and prevention

Yue-Zhang Wu

Chinese center for disease control and prevention

Cao Chen

Chinese center for disease control and prevention

Xiaoping Dong ( $\sim$ dongxp238@sina.com )

China CDC

Research article

Keywords: Prion, Scrapie infected mouse, Acetylation, Proteomics, KEEG pathway

Posted Date: May 18th, 2020

DOI: https://doi.org/10.21203/rs.3.rs-17989/v1

License: (c) (i) This work is licensed under a Creative Commons Attribution 4.0 International License.

Read Full License 


\section{Abstract}

\section{Background}

Acetylation is a reversible post-translational modification in eukaryotic and prokaryotic cells, actively participating in the regulation for biological functions and in the pathogenesis of diseases.

\section{Methods}

The acetylated proteins from the cortex regions of scrapie agents 139A- and ME7-infected mice collected at mid-early (80 days post-infection, dpi), mid-late (120 dpi) and terminal stage (180 dpi) were extracted. The global profiles of the brain acetylated proteins were assayed with proteomic mass spectrometry. The acetylated peptides whose levels were $>1.5$-fold higher or lower than that of age-matched normal controls were considered as differentially expressed acetylated peptides (DEAPs).

\section{Results}

A total of 1,485 acetylated peptides were identified. 118,42 and 51 DEAPs were in the brains of 139A-80 dpi, 120 dpi and $180 \mathrm{dpi}$, while 390, 227 and 75 DEAPs were in those of ME7-80 dpi, 120 dpi and $180 \mathrm{dpi}$, respectively. Overwhelming majority of the DEAPs in mid-early stage was down-regulated, while more portions of DEAPs in mid-late and late stage were up-regulated. Approximately $22.1 \%$ (328/1485) acetylated peptides were mitochondrial associated, which were mapped to 74 different proteins. Among them, 44 (59.5\%) proteins showed differentially expressed at least at one tested time-point. KEEG pathways analysis identified $39,13,10$ and $55,25,18$ pathways in the samples of 80,120 and 180 dpi of $139 \mathrm{~A}$ - and ME7-infected mice as significantly changed $(P<0.05)$, respectively. Six pathways were commonly involved in all tested samples, including carbon metabolism, metabolic pathways, biosynthesis of amino acids, glycolysis/gluconeogenesis, pyruvate metabolism and citrate cycle (TCA cycle). Moreover, dozens of steps in TAC cycle were affected via down-regulated acetylation for the relevant enzymes in the mid-early stage, while many steps were affected in the mid-late stage via upregulated acetylation. In the late stage, the affected steps focused on up-regulated acetylation for succinate dehydrogenase, fumarate hydratase and malate dehydrogenase.

\section{Conclusion}

Collectively, Our data here illustrated a picture of global acetylation for brain proteins during prion infection, showing remarkably inhibiting acetylation in the early stage and relatively enhanced acetylation in the late stage.

\section{Background}

Prion disease is a group of transmissible neurodegenerative disorders affecting a species of animals and humans [1]. The etiological agent is prion, a very unique pathogen without nucleic acid. Beside of the neuropathological hallmarks of prion disease, such as deposits of prions ( $\operatorname{PrPSC}^{\mathrm{Sc}}$, neuron loss and gliosis, 
numerous abnormalities of biological pathways in the central nerve system (CNS) have been identified, e.g., increased inflammatory reactions, activated autophagy, obvious apoptosis [2-5], which result from the aberrant alterations of the transcriptions and expressions of a large quantity of proteins and enzymes in the brains verified by the assays of transcriptomics and proteomics [6-9]. Moreover, the abnormal changes of post-translational modifications for brain proteins have been repeatedly described in the CNS tissues of various neurodegenerative diseases, involving in phosphorylation, S-nitrosylation, ubiquitylation, O-GIcNAcylation, acetylation, etc. [10-13].

Protein acetylation is a kind of important reversible protein modification in cells after translation process, which is regulated by acetylase and deacetylase $[14,15]$. This kind of post-translational modification participates actively in the regulation for protein functions, chromosome structure and the gene expressions. Recently, the developments and advances in mass spectrometry enable the localization of most acetylation sites in all proteomics at high resolution. Studies have proposed nearly 3,600 acetylation sites in about 1,750 proteins, suggesting that acetylation modification is one of the most abundant chemical modifications in nature [16-18]. In fact, acetylation may affect various physiological processes of proteins, including chromatin remodeling, cell cycle, splicing, nuclear transport, mitochondrial biology, and actin nucleation. In biology, acetylation plays an important role in immunity, circadian rhythm and memory formation. Even more, protein acetylation is a favorable target for the design of new drugs for various diseases.

The changes of acetylation for some proteins have been also reported in the brains of prion diseases. Previously, we have described that the levels and activities of Sirt1, a class III histone deacetylase, were remarkably decreased in the brains of different scrapie infected rodent models and in a prion infected cell line SMB-S15, resulting in increases of the acetylating forms of some Sirt1 target proteins, such as P53, PGC-1, and STAT3 [19]. Recently, we have also confirmed the declined levels of Sirt3 in the brain tissues of several scrapie infected mice and in SMB-S15 cells, leading to increases of acetylating forms of SOD2 and ATP $5 \beta$ that subsequently induce increase of intracellular ROS and reduction of ATP [20]. Aberrant alterations of acetylation modifications for other proteins have been also found in the brain tissues or the models in vitro of several neurodegenerative diseases, e.g., tau, microtubules, SOD1 [21-23]. However, the global change of protein acetylation in the brain tissues of prion diseases remains unsettled.

In this study, the brain samples collected at mid-early (80 days post-infection, dpi), mid-late (120 dpi) and terminal stage (about $180 \mathrm{dpi}$ ) from two different scrapie infected mouse models, strains 139A and ME7, were enrolled. $10 \%$ brain homogenates from three individual mice each infected model were pooled separately. After the processes of protein extraction, protein digestion and the peptide purification, the acetylated peptides were enriched and subjected into mass spectrometry. A total of 1,485 acetylated peptides were identified. Compared with the data of normal control, the peptides changing $>1.5$ fold were considered as the differentially expressed acetylated peptides (DEAPs). More numbers of DEAPs were identified in the samples of mid-early stage from both two scrapie infected mice, majority showing downregulated. The numbers of DEAPs were much less in the samples of mid-late and terminal stage and 
large portion of them converted to be up-regulated. Further, the mitochondrial associated DEAPs and the involved biological pathways were analyzed.

\section{Materials And Methods}

\section{Brain samples of scrapie infected experimental mice}

The brain samples of cortex regions from scrapie agents 139A- and ME7-infected mice collected at 80 , 120 and 180 dpi were enrolled into this study. Each group consisted of the tissues from three individual mice. The animal bioassays and confirmations of the experimental scrapie in the inoculated mice were described previously [24]. The brain samples of three age-matched normal mice each group were used as control.

\section{Western blot}

Brain tissues were homogenized in lysis buffer $(100 \mathrm{mM} \mathrm{NaCl}, 10 \mathrm{mM}$ EDTA, 0.5\% NP-40, 0.5\% sodium deoxycholate, $10 \mathrm{mM}$ Tris, $\mathrm{pH}$ 7.4) containing protease inhibitor cocktail set III. The homogenates were centrifuged at $2000 \times \mathrm{g}$ for $10 \mathrm{~min}$ and the supernatant fractions were collected. Aliquots of brain homogenate were separated by $12 \%$ SDS-PAGE and electroblotted onto nylon membranes. Membranes were blocked in TBS containing 5\% skimmed milk at room temperature (RT) for $2 \mathrm{~h}$ and incubated with various primary antibodies at $4{ }^{\circ} \mathrm{C}$ overnight, such as such as anti-PrP mAb (6D11, sc-58581; Santa Cruz Biotechnology), anti-GFAP (\#3670; Cell Signaling), anti-lba1 (sc32725;Santa Cruz Biotechnology), anti- $\beta$ actin antibody (1:5,000, huaxingbio, HX1827). After washing with TBS containing $0.1 \%$ Tween 20 (TBST), membranes were incubated with HRP conjugated secondary antibodies (Jackson ImmunoResearch Labs, 115-035-003 and 111-035-003) at RT for $1 \mathrm{~h}$. The blots were developed using enhanced chemiluminescence system (ECL, PerkinElmer, NEL103E001EA) and visualized on autoradiography films (General Electrics). Images were captured by ChemiDoc ${ }^{\mathrm{TM}}$ XRS + Imager and quantified by Image $\mathrm{J}$ software.

To detect the presence of proteinase K-resistant $\mathrm{PrPSc}^{\mathrm{Sc}}$, the brain homogenates or cell lysates were digested with a final concentration of $50 \mu \mathrm{g} / \mathrm{ml}$ proteinase $\mathrm{K}$ at $37^{\circ} \mathrm{C}$ for $1 \mathrm{~h}$ prior to Western blots.

\section{Protein extraction}

$500 \mathrm{mg}$ brain samples were homogenated by an automatic grinding machine in the lysis buffer ( $8 \mathrm{M}$ Urea, $30 \mathrm{mM}$ HEPES, $1 \mathrm{mM} \mathrm{Na}_{3} \mathrm{VO}_{4}, 2.5 \mathrm{mM} \mathrm{NA}_{3} \mathrm{PO}_{4}, 5 \mathrm{mM} \mathrm{C}_{4} \mathrm{H}_{7} \mathrm{NaO}_{2}$ ). The lysates were mixed with $10 \% \mathrm{TCA}-$ acetone and precipitated at $-20^{\circ} \mathrm{C}$ overnight. The precipitates were collected by centrifuging at $20000 \mathrm{rpm}$ for $30 \mathrm{~min}$ and washed with acetone for three times. After adding lysis buffer, the preparations were exposed to ultrasonic (pulse on $2 \mathrm{sec}$, pulse off $3 \mathrm{sec}$, power 180W) for $5 \mathrm{~min}$. Centrifugated at 20000 rpm for $30 \mathrm{~min}$, the supernatants were collected and incubated with $10 \mathrm{mM}$ DTT in final concentration at $56^{\circ} \mathrm{C}$ for $1 \mathrm{~h}$. The products were immediately mixed with $55 \mathrm{mM}$ IAM in final concentration and stayed in dark for $1 \mathrm{~h}$. The protein concentrations in the products were measured with Bradford protein assay. The 
quality of the extracted proteins was evaluated by SDS-PAGE, which showed clear bands (Supp Fig 1A). The protein quantitative standard curve showed R2 value was 0.9932 that fulfilled the requirement of the SOP of experiment.

\section{Protein digestion and peptide purification}

Aliquots of $10 \mathrm{mg}$ proteins each preparation were transferred into a $10 \mathrm{~K}$ ultrafilter and centrifuged at $4000 \mathrm{rpm}$ at $4^{\circ} \mathrm{C}$ for $40 \mathrm{~min}$. After discarding the waste liquid, $5 \mathrm{ml}$ of $5 \mathrm{mM}$ TEBA was added into the filters and sped at $4000 \mathrm{rpm}$ at $4^{\circ} \mathrm{C}$ for $40 \mathrm{~min}$. Above procedures were repeated again. The protein digestion was performed with $1 \mu \mathrm{g} / \mu \mathrm{l}$ Trypsin at $37^{\circ} \mathrm{C}$ for $24 \mathrm{~h}$. The purification of peptides was conducted with $\mathrm{C} 18$ reversed-phase column in order to remove salts and dried by vacuum. Measurement of the digested peptides showed the lengths of the majority were less than 30 amino acids (Supp Fig 1B).

\section{Enrichment of the acetylated peptides}

The dried samples were solved in IAP buffer containing $50 \mathrm{mM} \mathrm{MOPS} \mathrm{pH7.2,} 10 \mathrm{mM} \mathrm{Na}_{3} \mathrm{PO}_{4}$ and $50 \mathrm{mM}$ $\mathrm{NaCl}$ and pellets were discarded by centrifuged at $10000 \mathrm{rpm}$ at $4^{\circ} \mathrm{C}$ for $5 \mathrm{~min}$. The enrichment of the acetylated peptides was conducted with a commercial kit according to the manufacturer's instruction (ICP0388, ImmuneChem Pharmaceuticals). After washing the beads conjugated with acetylated antibody with PBS, $2 \%$ acetic acid and IAP buffer, the samples were added and incubated overnight rotatably. The beads were collected by low speed centrifuge and washed with IPA buffer and dd water.

\section{Peptide labelling and identification of mass spectra}

The enriched acetylated peptides were mixed with $41 \mu$ acetonitrile, vortexed for 1 min and pelleted after short sped. Different isotopes were added into the tested peptide preparations and maintained at RT for 1 $\mathrm{h}$, and then, mixed with $8 \mu \mathrm{l}$ hydroxylamine and maintained for $15 \mathrm{~min}$. The labeled products were further dried by vacuum.

Desalted peptide mixture were loaded onto a Acclaim PePmap C18-reversed phase column $(75 \mu \mathrm{m} \times 2 \mathrm{~cm}$, $3 \mu \mathrm{m}, 100 \AA$ Á, Thermo Scientific) and separated with reversed phase C18 column $(75 \mu \mathrm{m} \times 10 \mathrm{~cm}, 5 \mu \mathrm{m}$, 300 Á, Agela Technologies) mounted on a Dionex ultimate 3000 nano LC system. Peptides were eluted using a gradient of $5-80 \%(\mathrm{v} / \mathrm{v})$ acetonitrile in $0.1 \%$ formic acid over $45 \mathrm{~min}$ at a flow rate of $300 \mathrm{~nL}$ min-1 combined with a Q Exactive mass spectrometer (Thermo Fisher Scientific, MA, USA).

The eluates were directly entered Q-Exactive MS (Thermo Fisher Scientific, Waltham, MA, USA), setting in positive ion mode and data-dependent manner with full MS scan from 350-2000 m/z, full scan resolution at $70000, \mathrm{MS} / \mathrm{MS}$ scan resolution at 35,000 . MS/MS scan with minimum signal threshold $1^{\mathrm{E}+5}$, isolation width at $2 \mathrm{Da}$. To evaluate the performance of this mass spectrometry on the ITRAQ labeled samples, two MS/MS acquisition modes, higher collision energy dissociation (HCD) was employed. To optimize the MS/MS acquisition efficiency of HCD, normalized collision energy (NCE) was systemically examined 30, stepped $20 \%$. 
Quantitative analysis of immunoblot images was carried out using software Image J. The gray values of each target blot were evaluated. Quantitative evaluations of fluorescent intensity in IFA assays were automatically performed with the software Columbus in Operetta. Statistical analyses were performed using Student's $t$ test.

\section{Results}

Aberrant alterations of PrPSC, GFAP and Iba 1 in the brain samples of 139A-and ME7-infected mice collected at mid-early, mid-late and terminal stages

The clinical, neuropathological and pathogenic features of scrapie agents 139A- and ME7-infected C57 mice were thoroughly described previously. The mean incubation times of the mice infected with agents 139A and ME7 were $183.9 \pm 23.1$ (154-226) and $184.2 \pm 11.8$ (165-193) days, respectively [24]. To test the changes of main hallmarks of prion disease in the brain samples of scrapie infected mice collected at mid-early, mid-late and terminal stages, which were employed into further proteomic assays, the levels of PrPSc, GFAP and Iba1 were evaluated by the individual specific Western blots. PK-resistant PrP signals (PrPres or $\mathrm{PrPS}^{\mathrm{Sc}}$ ) were detected in all brain samples of scrapie infected mice, showing three bands ranging from 20-30 kDa (Fig 1A). Quantitative assay of the signal intensities showed that the PrPSc in the samples of mid-early stage were weak and became much stronger in those of mid-late and late stage (Fig 1A). As expected, the signals of GFAP (Fig 1B) and Iba1 (Fig 1C) in the brain samples of the scrapie infected mice displayed time-dependent increased, while maintained almost unchanged in the brain tissues of normal mice (data not shown).

Global acetylated proteins profiles of the brain samples of 139A-and ME7-infected mice collected at the mid-early, mid-late and terminal stages

The homogenates of cortex regions of three individual mice infected with scrapie agents 139A and ME7 collected at mid-early, mid-late and final stages were pooled as the samples of 139A-80 dpi, 139A-120 dpi, 139A-180 dpi, as well as ME7-80 dpi, ME7-120 dpi, ME7-180 dpi, respectively. Meanwhile, the homogenates of cortex regions of three age-matched individual normal mice were pooled as the normal control. The quality of extracted proteins, quantitative accuracy of proteins, trypsin hydrolysis efficiency, mass spectral mass deviation, mass spectrometry acquisition intensity and data volume fulfilled the requirements of the quality control for proteomic assays. Totally, 271,383 MS/MS spectra were achieved and 63,365 of them were matched spectra. 2,250 proteins, elicited from 7,831 unique peptides, were identified with $95 \%$ confidence interval by the Peptide Prophet Algorithm. A total of 1,485 acetylated peptides were identified.

The DEAPs in the brains between scrapie infected and normal mice were calculated. Out of 1,485 acetylated peptides, the DEAPs (changing $>1.5$ fold) in the brains of 139A-80 dpi, $139 \mathrm{~A}-120 \mathrm{dpi}$ and $139 \mathrm{~A}-$ 180 dpi were 118 (7.9\%), 42 (2.8\%) and 51 (3.4\%), while those in the brains of ME7-80 dpi, ME7-120 dpi 
and ME7-180 dpi were 390 (26.3\%), 227 (15.3\%) and 75 (5.1\%), respectively (Fig 2A). The DEAPs (changing $>2.0$ fold) in the brains of 139A-80 dpi, 139A-120 dpi and 139A-180 dpi were 8 (0.54\%), 5 $(0.34 \%)$ and $6(0.4 \%)$, while those in the brains of ME7-80 dpi, ME7-120 dpi and ME7-180 dpi were 12 $(0.81 \%), 36(2.4 \%)$ and $91(6.1 \%)$, respectively (Fig 2B). Obviously, much more decreased DEAPs in the brain samples of the early stage, while more increased DEAPs in the mid-late and late stages of 139Aand ME7-infected mice were identified both in the groups of $>1.5$ fold and $>2.0$ fold (Fig 2). Additionally, more DEAPs were found in the brains of ME7-infected mice at every time-point than those of 139Ainfected mice. For exploring the potential changes in proteomic profiles among the different brain samples maximally, we used the peptides that were $>1.5$-fold increased or decreased as DEAPs in this study.

The profiles of DEAPs in mitochondria of the brain samples of 139A-and ME7-infected mice collected at the mid-early, mid-late and terminal stages

Out of 1,485 identified acetylated peptides, 328 peptides were marked as mitochondrial OS, which accounting for $22.1 \%$ of total acetylated peptides. 135 acetylated peptides $(41.1 \%)$ showed differentially expressed ( $>1.5$-fold increased and/or $<0.666$-fold decreased) in one or more tested samples of scrapie infected mice. The numbers of DEAPs in the samples each time-point of those two scrapie infected mice were illustrated in Fig 3A. Similar as the global profile of DEAPs, the numbers of DEAPs in the brain samples of mid-early stages of scrapie infected mice were much more than those of mid-late and terminal stages. More DEAPs were observed in the samples of ME7 than 139A infected mice. In the samples of $80 \mathrm{dpi}, 99$ (98\%) out of 101 DEAPs in ME7 and 30 (96.8\%) out of 31 DEAPs in 139A were down-regulated ones. In the samples of $120 \mathrm{dpi}$, the decreased DEAPs reduced to $15.9 \%$ (7/44) in ME7 and $54.5 \%(6 / 11)$ in 139A. In the samples of late stage (180 dpi), all 4 DEAPs in 139A were increased ones, while $3(20 \%)$ out of 15 DEAPs in ME7 showed increased.

Further, the consistency and dissimilarity of the mitochondrial associated DEAPs each time-point between two scrapie infected mice were analyzed. The acetylated peptides showing differentially expressed in both those two scrapie experimental mice were considered as overlapped ones. The acetylated peptides showing differentially expressed in one infected model, but not differentially expressed while expressed at the same tendency $(>1.5$ and $<1.0$ for the increased ones and $>1.0$ and $<0.666$ for the decreased ones) in another model were considered as consistent ones. The acetylated peptides showing differentially expressed in one infected model, whereas expressed at the opposite tendency were considered as the dissimilar ones. As shown in Fig 3B, in the samples of mid-early stage, 29 acetylated peptides were overlapped, 73 were consistent and none was dissimilar between 139A and ME7 infected mice. In the samples of mid-late stage, 6 were overlapped, 37 were consistent and 4 were dissimilar. In the samples of late stage, none was overlapped, but 17 were consistent and only one was dissimilar. It indicates that predominantly majority of the identified acetylated mitochondrial associated peptides were consistently expressed in the brains of those two kinds of scrapie infected mice at three time-points during infection, although the samples of ME7-infected mice contains much more DEAPs than 139A-infected ones. 
Bioinformatic assay revealed that 328 identified acetylated mitochondrial associated peptides belonged to 74 different proteins, among them $44(59.5 \%)$ proteins showed differentially expressed at least at one tested time-point. The names of those 44 proteins, as well as the numbers of the identified peptides, the numbers of DEAPs, the acetylated positions of the individual differentially expressed proteins and the DEAPs in the tested samples were summarized in Table 1. 9 (20.5\%) out of 44 proteins contained 10 or more than 10 identified peptides, including aconitate hydratase (with 6 DEAPs), aspartate aminotransferase (with 14 DEAPs), ATP synthase subunit alpha (with 8 DEAPs), ATP synthase subunit beta (with 5 DEAPs), dihydrolipoyl dehydrogenase (with 6 DEAPs), glutamate dehydrogenase 1 (with DEAPs), isocitrate dehydrogenase [NADP] (with 2 DEAPs), malate dehydrogenase (with 8 DEAPs), pyruvate dehydrogenase E1 component subunit alpha (with 8 DEAPs). In addition, 24 (54.5\%) differentially changed proteins contained 2 to 9 identified peptides and $11(25 \%)$ proteins contained only one identified peptide.

Only three up-regulated DEAPs were identified in the samples of mid-early stage, two in ME7- and one in 139A-infected mice. All three up-regulated DEAPs belonged to ATP synthase subunit alpha, and the acetylated sites were at aa. 194 (for both ME7 and 139A) and aa. 541 (for ME7), respectively. Further analysis revealed that the level of the acetylated peptide at aa.541 in the sample of 139A-80 dpi was 1.4675 -fold increased. Those two acetylated peptides turned to the normal ranges in the samples of 120 and $180 \mathrm{dpi}$ of both ME7- and 139A-infected mice. 7 down-regulated DEAPs in the samples of $80 \mathrm{dpi}$ changed to significantly up-regulated in $120 \mathrm{dpi}$. They were acyl-coenzyme A thioesterase 2 acetylated at aa.83, acyl-coenzyme A thioesterase 9 acetylated at aa.102, dihydrolipoyl dehydrogenase acetylated at aa.122, dihydrolipoyllysine-residue succinyltransferase component of 2-oxoglutarate dehydrogenase complex acetylated at aa.268, isocitrate dehydrogenase [NAD] subunit alpha acetylated at aa.200 and aa.214, peptidyl-prolyl cis-trans isomerase $\mathrm{F}$ acetylated at aa.85, pyruvate dehydrogenase $\mathrm{E} 1$ component subunit alpha acetylated at aa.244 and 313, succinate-CoA ligase [ADP-forming] subunit beta acetylated at aa.88. In parallel, 79 down-regulated DEAPs in the samples of $80 \mathrm{dpi}$ turned to normal ranges in that of 120 and $180 \mathrm{dpi}$, majority showing increasing trends in $120 \mathrm{dpi}$ and decreasing slightly in $180 \mathrm{dpi}$. Only 12 down-regulated DEAPs in the samples of $80 \mathrm{dpi}$ maintained still significantly down-regulated in the subsequent samples. In the samples of $120 \mathrm{dpi}, 26$ DEAPs showed up-regulated only in this time-point, while 7 up-regulated ones were down-regulated significantly in $80 \mathrm{dpi}$ and 6 up-regulated ones maintained in the increased status in $180 \mathrm{dpi}$. In addition, all 11 down-regulated and 7 up-regulated DEAPs in the samples of $180 \mathrm{dpi}$ were also identified as DEAPs in those of 80 and/or $120 \mathrm{dpi}$. Those data indicate that during scrapie infection, the acetylation of the mitochondrial related proteins in the whole brain tissues is markedly down-regulated in the mid-early stage, become active in the mid-late stage and turn to relatively silent in the terminal stage.

Involvement of the significant pathways in the brain samples of 139A-and ME7-infected mice collected at the mid-early, mid-late and terminal stages

To analyze the possible involved biological pathway of the DEAPs, Kyoto Encycloppedia of Genes and Genomes pathway analysis was conducted using the software of KOBAS2.0. As shown in Fig 4A, 55, 25, 
18 and 39, 13, 10 biological pathways were significantly changed $(P<0.05)$ in the samples of 80,120 and $180 \mathrm{dpi}$ of ME7- and 139A-infected mice, respectively. The top 10 involved pathways based on their $\mathrm{P}$ values and the numbers of the differentially expressed acetylated proteins each tested samples were summarized in Table 2. Six significantly changed biological pathways were identified in all tested samples, including Carbon metabolism, Metabolic pathways, Biosynthesis of amino acids, Glycolysis/Gluconeogenesis, Pyruvate metabolism and Citrate cycle (TCA cycle). Subsequently, the numbers of the involved up- and down-regulated DEAPs of those six pathways at each tested sample were counted (Fig 4B-G). All six pathway showed the similar distributing patterns of DEAPs, with absolutely predominantly down-regulated DEAPs in the mid-early stage and up-regulated DEAPs in the mid-late and late stage. Meanwhile, the numbers of the involved DEAPs in those pathways reduced along with the incubation, particularly in the brain samples of ME7-infected mice. Two kinds of scrapie infected mice models also displayed other differences beside of the numbers of the involved DEAPs. In the samples of 139A-infected mice, all involved DEAPs in the six pathways were down-regulated in $80 \mathrm{dpi}$ and up-regulated in $180 \mathrm{dpi}$. In the samples of $120 \mathrm{dpi}$, the DEAPs in three pathways were all upregulated, while the other three ones contained only one down-regulated DEAP. Similar pattern was also observed in the samples of ME7-infected mice, overwhelming majority of down-regulated DEAPs in that of $80 \mathrm{dpi}$ and majority of up-regulated DEAPs in $120 \mathrm{dpi}$. In the samples of $180 \mathrm{dpi}$, the numbers of DEAPs were much less and the ratios of up- and down-regulated DEAPs in the most (5/6) involved pathways were almost same.

Changes of the involved DEAPs in the pathway of citrate cycle (TCA cycle) in the brain samples of $139 \mathrm{~A}$ and ME7-infected mice collected at the mid-early, mid-late and terminal stages

Citrate cycle or TCA cycle is an essential metabolism pathway in the aerobic body, which locates in cytoplasm of prokaryote and mitochondria of eukaryote. The acetylation processes of dozens of enzymes in TCA cycles showed significantly changed in the brain tissues during prion infections, covering 13 and 8 down-regulated DEAPs in $80 \mathrm{dpi}, 8$ and 2 up-regulated ones in $120 \mathrm{dpi}, 2$ and 2 upregulated ones in $180 \mathrm{dpi}$ of ME7- and 139A-infected mice, respectively (Fig 4C). Furthermore, the status of each DEAP at those three time-points were analyzed (Table 3). 7 out of 8 down-regulated DEAPs in 80 dpi of 139A-infected mice were also significantly decreased in that of ME7-infected mice. The rest of the involved DEAPs observed only in one type of scrapie infected mouse (5 in ME7 and 1 in 139A) also showed down-regulated tendencies in another model despite of insignificance. Most of those downregulated DEAPs turned to raise in $120 \mathrm{dpi}$, among them 4 DEAPs were even significantly up-regulated. All down-regulated DEAPs in 80 dpi dropped down to the normal ranges in $180 \mathrm{dpi}$. All eight DEAPs in TCA cycle identified in 120 dpi were up-regulated ones, among them, two DEAPs were commonly detected in both 139A-and ME7-infected mice. 3 out of 8 up-regulated DEAPs in 120 dpi still maintained as increased ones, while other 5 ones turned to normal ranges in $180 \mathrm{dpi}$. In the samples of $180 \mathrm{dpi}$, only 4 DEAPs were identified in TCA cycle, all of them being up-regulated. Those data strongly indicate that the acetylating processes of the enzymes of TCA cycle in the brain tissues were negatively regulated in the early stage and positively regulated in the mid-late and terminal stage of prion infection. 
The potential affected steps in TCA cycle due to the changes of DEAPs in the brain tissues at different time-points during prion infection were evaluated. Dozens of steps in TAC cycle were affected via negatively regulated acetylation for the relevant enzymes in the samples of $80 \mathrm{dpi}$ (left panels in Fig $5 \mathrm{~A}$ and B). Many steps were also affected in $120 \mathrm{dpi}$ via up-regulated acetylation for the enzymes, particularly in ME7-infected mice (middle panels in Fig 5A and B). In the samples of $180 \mathrm{dpi}$, the affected steps focused on the processes from succinate to fumarate, fumarate to malate and malate to oxaloacetate, via positively regulated acetylation for succinate dehydrogenase, fumarate hydratase and malate dehydrogenase (right panels in Fig $5 \mathrm{~A}$ and B). It indicates that the activity of TCA cycle in the context of whole brain is strongly influenced by deacetylation in the early stage and by acetylation in the mid-late and late stage during prion infection.

\section{Discussion}

In this study, we have screened the global acetylation situations of the brain proteins in two scrapie infected mouse models at mid-early, mid-late and terminal stages using advanced proteomic technique. The potential involved biological functions and pathways due to the alterations of the protein acetylation have been analyzed. Totally, 1,485 acetylated peptides in the brain tissues of mouse have been identified and hundreds of them shows differentially expressed, either 1.5-fold increased or decreased, at various time-points during scrapie infection. To our knowledge, this is the first comprehensive description for the global protein acetylation in the brain tissues during prion infection.

Two scrapie infected mouse models show the similar alterative patterns of the global acetylating profiles of the whole brain proteins during infection, despite of the difference in the numbers of DEAPs. In the brains of the mid-early stage ( $80 \mathrm{dpi}$ ) there are markedly more numbers of DEAPs and the absolutely majority display down-regulated. In the mid-late (120 dpi) and terminal ( $180 \mathrm{dpi})$ stage, the numbers of DEAPs reduce and large portions of them are up-regulated. The alterative tendencies of the brain protein acetylating profiles during prion infection are completely different from the changes of global profiles of brain proteins in those two scrapie infected mouse models [8]. In that proteomic study, much more numbers of the differentially expressed proteins (DEPs) are observed in the samples of terminal stage (180 dpi) than in that of mid-late stage (80 dpi) [8]. It implies that the brain protein acetylation situations are reversible during prion infection.

As expected, majority of DEAPs during prion infection involves in the enzymatic proteins participating in the various metabolism activities. The analyses of the involved KEEG pathways also reveal the same feature. Large portions of the involved pathways are associated with the metabolism, e.g., carbon metabolism, metabolic pathways, glyoxylate and dicarboxylate metabolism, pyruvate metabolism, TCA cycle, etc. However, in the assays of global brain proteomics for 139A-and ME7-infected mice [8] and for different subtypes of human prion diseases [6], the most frequently involved pathways are the ones related with inflammation, e.g., complement and coagulation cascades, Staphylococcus aureus infection, Chagas disease (American trypanosomiasis), Leishmaniasis, systemic lupus erythematosus, etc., in which up-regulations of some complement components (C1q, C2, C3, and C4) are commonly addressed. 
The diversity of the involved pathways addressed by two types of proteomic assays reflects two different aspects of the whole proteins in the brains infected with prions.

Mitochondria are the sites of oxidative metabolism in eukaryotes, where sugars, fats and amino acids eventually oxidize and release energy [25]. Our data here have proposed that approximately $1 / 3$ identified acetylated peptides are marked as mitochondrial OS and more than $40 \%$ mitochondrial associated acetylated peptides are DEAPs. Despite of the difference in the numbers of DEAPs, two scrapie infected mouse models show the similar alterative patterns of mitochondrial associated DEAPs during the infection, that is the vast majority of down-regulated DEAPs in the mid-early stage but more ratios of upregulated DEAPs in the mid-late and terminal stage. Moreover, overwhelming majority of the identified brain mitochondrial associated DEAPs in those two scrapie mouse models also show the same changing tendency during the infection. The mitochondrial associated DEAPs subsequently induce the significant changes of a series of pathways. Six significantly changed pathways identified in all tested samples contain the processes within mitochondria. Obviously, the brain mitochondrial functions are influenced by protein deacetylation (in the early and mid-early stage) and acetylation (in the mid-late and terminal stage) during prion infection.

TCA cycle is an essential metabolic pathway in aerobic organisms. It is the final metabolic pathway of the three major nutrients, as well as the junction of the metabolism of sugars, lipids and amino acids [26]. TCA cycle is carried out within the matrix of the mitochondrion, which contains eight enzymatic steps including citrate synthase, aconitate hydratase, isocitrate dehydrogenase, oxoglutarate dehydrogenase, succinate-CoA ligase, succinate dehydrogenase, fumarate hydratase and malate dehydrogenase [27]. In the mid-early stage, all eight enzymes are in the situation of deacetylation. However, in the mid-late stage, six enzymes (isocitrate dehydrogenase, oxoglutarate dehydrogenase, succinate-CoA ligase, succinate dehydrogenase, fumarate hydratase and malate dehydrogenase) in the sample of ME7 infected mice and one (oxoglutarate dehydrogenase) in 139A-infected ones are significantly acetylated, while the rests are in the normal level. In the late stage, three enzymes (succinate dehydrogenase, fumarate hydratase and malate dehydrogenase) in ME7 infected mice and two (fumarate hydratase and malate dehydrogenase) in 139A infected ones are in the status of acetylation. The alteration and reversion of the acetylation and deacetylation of almost all enzymes in TCA cycle may reflect the change of the activity of this pathway in the brains during prion infection. As the core process linked with the metabolism of carbohydrates, fats and proteins $[27,28]$, changes of TCA cycle will definitely affect the other metabolic pathways.

Although histone acetylation has been demonstrated to play a critical role in chromatin remodeling for long time [29-31], the functional roles of the acetylation of the vast majority of thousands of non-histone proteins are still nuclear. Advances in mass spectrometry-based proteomics have proposed that a vast of the acetylated proteins involve in metabolic pathways both in eukaryotes and bacteria, such as glycolysis and TCA cycle [16, 32]. By performing a proteomic analysis of 48 phylogenetically distant bacteria, Choudhary and the colleagues have reported that lysine acetylation occurs in evolutionarily conserved lysine residues in catalytic sites of enzymes involved in central carbon metabolism and plays a direct role in metabolic regulation by switching off enzyme activity [33]. ApoE-deficient C57BI/6J mice exposed to 
low-dose-rate gamma radiation shows that the great majority of the hyperacetylated proteins are involved in the TCA cycle, fatty acid oxidation, oxidative stress response and sirtuin pathway, which is associated with impairment of heart metabolism [34]. Although it still lacks the direct evidence to support the association of enzymic acetylation with the functions of metabolic pathways in the brains of prion diseases, it is reasonable to speculate that hyperacetylation of enzymes in TCA cycle might be a negative regulator for enzymatic activity whereas deacetylation might benefit for TCA cycle. Further assays addressing the association between acetylation status and enzymatic activity in metabolic pathways, e.g., TCA cycle, during prion infection are mostly deserved.

The process of protein acetylation and deacetylation is a reversible post-translation modification in regulating cellular function and response to external stimuli $[15,35]$. The data here also illustrate the reversible alteration of brain protein acetylation during prion infection. However, we have to admit that the changes of protein acetylation in this study reflect the alteration in the context of whole brain tissues. Similar changing profiles are also observed in the brain levels of nitric oxide, nitric oxide synthase and the Snitrosylation of several proteins, e.g., aquaporin-1, calcium/calmodulin-dependent protein kinase II, neurogranin and opalin in several experimental scrapie infected rodents [36]. The exact reason for the reversible alteration of brain protein acetylation during prion infection is unclear. One possibility is the association with the changes of deacetylases, such as sirtuin pathway, in the brains during prion infection. Our previous studies have showed that besides of marked declines of brain Sirt1 and Sirt3 at the terminal stage of different prion diseases, the brain level of Sirt 1 in the scrapie agent $263 \mathrm{~K}$-infected hamsters maintains at high level in the early stage, starts to drop down in the mid-late stage and continues to decrease till the end stage [19]. Another possibility is related with the gradual appearances of neuropathological abnormalities, such as $\mathrm{PrPSc}$ deposit, astrogliosis and activated microglia in the brain tissues. Our data here and in other previous studies $[8,37,38]$ demonstrate that the amounts of $\mathrm{PrPS}^{\mathrm{Sc}}$ in the samples of mid-early stage ( $\left.80 \mathrm{dpi}\right)$ are remarkably less than in that of mid-late $(120 \mathrm{dpi})$ and end (180 dpi) stage of those two scrapie infected mouse models. Meanwhile, significant increases of GFAP- and Iba1-signals are also detected since mid-late stage. Notably, the marked neuropathological changes occur since mid-late stage. In that case, we may assume that in the early stage of prion infection with less $\mathrm{PrPSc}^{\mathrm{Sc}}$ deposit and reactive gliosis, the host brain is still capable of maintaining the biological functions in order to overcome the neurotoxicity produced by prion infection, at least partially, via deacetylation for the enzymes associated with various metabolic pathways. Along with the more PrPSc deposit and severe reactive inflammation in the CNS tissues in the late stage, the deacetylation process may be inhibited, for example, due to the decline of brain levels of sirtuin pathway, which is likely to contributes to the impairment of mitochondrial function.

In addition, evaluation of the protein acetylation during prion infection in this study is the global changes of whole brain tissues that contain not only the damaged neurons but also the proliferative astrocytes and activated microglia. We cannot simply conclude whether such alteration is due to the accumulation of aggregated proteins or due to the subsequently reactive inflammation. We are also unable to 
distinguish the defined changes of protein acetylation in the damaged neurons, the proliferative astrocytes and the activated microglia. Those questions deserve further exploration.

\section{Conclusions}

In conclusion, our data here illustrated a picture of global acetylation for brain proteins during prion infection, showing remarkably inhibiting acetylation in the early stage and relatively enhanced acetylation in the late stage. And in the late stage, the affected steps focused on up-regulated acetylation for succinate dehydrogenase, fumarate hydratase and malate dehydrogenase. It possibly reflects host (brain) response to external prions in order to overcome the neurotoxicity in the early stage and the impairment of metabolic and mitochondrial functions due to $\mathrm{PrPSc}^{\mathrm{Sc}}$ deposit and reactive inflammation.

\section{Abbreviations}

ADP adenosine diphosphate

ATP adenosine Triphosphate

ATP5 $\beta$ adenosine Triphosphate synthase subunit beta

CNS central nerve system

DEAPs differentially expressed acetylated peptides

DEPs differentially expressed proteins

Dpi post-infection

GFAP Glial fibrillary acidic protein

Iba1 lonized calcium binding adaptor molecule 1

KEEG Kyoto Encyclopedia of Genes and Genomes

NADP nicotinamide adenine dinucleotide phosphate

PGC-1 peroxisome proliferator-activated receptorycoactivator-1

PrP Prion protein

$\mathrm{PrP}^{\mathrm{C}}$ cellular prion protein

$\operatorname{PrP}^{\mathrm{Sc}}$ Scrapie associated prion protein

ROS reactive oxygen species 
Sirt1 sirtuin 1

Sirt3 sirtuin3

SOD1 manganese superoxide dismutase, superoxide dismutase 1

SOD2 manganese superoxide dismutase, superoxide dismutase 2

STAT signal Transducer and Activator of Transcription

TCA cycle tricarboxylic acid cycle

\section{Declarations}

\section{Ethics approval}

All procedures involving live animals were approved by the Ethics Committee of National Institute for Viral Disease Control and Prevention. All protocols were designed to minimize animal discomfort. The approval identifications for this study were animal use document 2013031.

\section{Consent for publication}

Not applicable.

\section{Availability of data and materials}

The data and materials are available from corresponding author on reasonable request.

\section{Competing interests}

The authors declare that they have no competing interests.

\section{Funding}

This work was supported by Chinese National Natural Science Foundation Grants (81630062), National Key R\&D Program of China (2017YFC1200500®2018ZX10711001) and Grant(No.2019SKLID501区 2019SKLID603 $₫ 2019 S K L I D 307)$ from the State Key Laboratory for Infectious Disease Prevention and Control (China CDC).

\section{Authors' contributions}

Q.S. contributed to study design, data analysis, and prepared the manuscript. D.D.C. and Adalaiti assisted with study design and performed the assays of Western Blot analysis. K.X and Y.Z.W assisted with the preparation of the experimental animal samples.L.P.G. and X.H.Y and C.C assisted with statisticalanalysis. X.P.D., corresponding authors, contributed to design, study concept, and final manuscript preparation.All authors read and approved the final manuscript. 


\section{Acknowledgements}

Not applicable.

\section{References}

1. Chen, C. and X.P. Dong, Epidemiological characteristics of human prion diseases. Infect Dis Poverty, 2016. 5(1): p. 47.

2. Shi, Q., et al., Brain microglia were activated in sporadic CJD but almost unchanged in fatal familial insomnia and G114V genetic CJD. Virol J, 2013. 10: p. 216.

3. Lv, Y., et al., Remarkable Activation of the Complement System and Aberrant Neuronal Localization of the Membrane Attack Complex in the Brain Tissues of Scrapie-Infected Rodents. Mol Neurobiol, 2015. 52(3): p. 1165-1179.

4. Xu, Y., et al., FBXW7-Induced MTOR Degradation Forces Autophagy to Counteract Persistent Prion Infection. Mol Neurobiol, 2016. 53(1): p. 706-719.

5. Xu, Y., et al., Activation of the macroautophagic system in scrapie-infected experimental animals and human genetic prion diseases. Autophagy, 2012. 8(11): p. 1604-20.

6. Shi, Q., et al., Proteomics analyses for the global proteins in the brain tissues of different human prion diseases. Mol Cell Proteomics, 2015. 14(4): p. 854-69.

7. Tian, C., et al., Global transcriptional profiling of the postmortem brain of a patient with $\mathrm{G} 114 \mathrm{~V}$ genetic Creutzfeldt-Jakob disease. Int J Mol Med, 2013. 31(3): p. 676-88.

8. Shi, Q., et al., Comparative proteomics analyses for 139A and ME7 scrapie infected mice brains in the middle and terminal stages. Proteomics Clin Appl, 2017. 11(5-6).

9. Voet, S., et al., Inflammasomes in neuroinflammatory and neurodegenerative diseases. EMBO Mol Med, 2019. 11(6).

10. Chen, L.N., et al., Proteomic Analyses for the Global S-Nitrosylated Proteins in the Brain Tissues of Different Human Prion Diseases. Mol Neurobiol, 2016. 53(8): p. 5079-96.

11. Katsumoto, A., H. Takeuchi, and F. Tanaka, Tau Pathology in Chronic Traumatic Encephalopathy and Alzheimer's Disease: Similarities and Differences. Front Neurol, 2019. 10: p. 980.

12. Alonso, A.D., et al., Hyperphosphorylation of Tau Associates With Changes in Its Function Beyond Microtubule Stability. Front Cell Neurosci, 2018. 12: p. 338.

13. Ansari, S.A. and B.S. Emerald, The Role of Insulin Resistance and Protein O-GlcNAcylation in Neurodegeneration. Front Neurosci, 2019. 13: p. 473.

14. Sterner, D.E. and S.L. Berger, Acetylation of histones and transcription-related factors. Microbiol Mol Biol Rev, 2000. 64(2): p. 435-59.

15. Yang, X.J. and E. Seto, Lysine acetylation: codified crosstalk with other posttranslational modifications. Mol Cell, 2008. 31(4): p. 449-61. 
16. Choudhary, C., et al., Lysine acetylation targets protein complexes and co-regulates major cellular functions. Science, 2009. 325(5942): p. 834-40.

17. Deng, W., et al., GPS-PAIL: prediction of lysine acety/transferase-specific modification sites from protein sequences. Sci Rep, 2016. 6: p. 39787.

18. Carrico, C., et al., The Mitochondrial Acylome Emerges: Proteomics, Regulation by Sirtuins, and Metabolic and Disease Implications. Cell Metab, 2018. 27(3): p. 497-512.

19. Wang, J., et al., Scrapie infection in experimental rodents and SMB-S15 cells decreased the brain endogenous levels and activities of Sirt1. J Mol Neurosci, 2015. 55(4): p. 1022-30.

20. Maimaitiming, A., et al., Aberrant Decrease of the Endogenous SIRT3 and Increases of Acetylated Proteins in Scrapie-Infected Cell Line SMB-S15 and in the Brains of Experimental Mice. ACS Chem Neurosci, 2019. 10(10): p. 4293-4302.

21. Sonawane, S.K. and S. Chinnathambi, Prion-Like Propagation of Post-Translationally Modified Tau in Alzheimer's Disease: A Hypothesis. J Mol Neurosci, 2018. 65(4): p. 480-490.

22. Phadwal, K., et al., Spermine increases acetylation of tubulins and facilitates autophagic degradation of prion aggregates. Sci Rep, 2018. 8(1): p. 10004.

23. Rasouli, S., et al., Lysine acylation in superoxide dismutase-1 electrostatically inhibits formation of fibrils with prion-like seeding. J Biol Chem, 2017. 292(47): p. 19366-19380.

24. Xiao, K., et al., Re-infection of the prion from the scrapieinfected cell line SMB-S15 in three strains of mice, CD1, C57BL/6 and Balb/c. Int J Mol Med, 2016. 37(3): p. 716-26.

25. Glancy B. Visualizing Mitochondrial Form and Function within the Cell. Trends Mol Med. 2020. 26(1):p. 58-70.

26. Murphy, M.P. and L.A.J. O'Neill, Krebs Cycle Reimagined: The Emerging Roles of Succinate and Itaconate as Signal Transducers. Cell, 2018. 174(4): p. 780-784.

27. van der Bliek, A.M., M.M. Sedensky, and P.G. Morgan, Cell Biology of the Mitochondrion. Genetics, 2017. 207(3): p. 843-871.

28. Altman, B.J., Z.E. Stine, and C.V. Dang, From Krebs to clinic: glutamine metabolism to cancer therapy. Nat Rev Cancer, 2016. 16(10): p. 619-34.

29. Hebbes, T.R., A.W. Thorne, and C. Crane-Robinson, A direct link between core histone acetylation and transcriptionally active chromatin. EMBO J, 1988. 7(5): p. 1395-402.

30. Cabot, B. and R.A. Cabot, Chromatin remodeling in mammalian embryos. Reproduction, 2018. 155(3): p. R147-R158.

31. Kim, S. and B.K. Kaang, Epigenetic regulation and chromatin remodeling in learning and memory. Exp Mol Med, 2017. 49(1): p. e281.

32. Zhao, S., et al., Regulation of cellular metabolism by protein lysine acetylation. Science, 2010. 327(5968): p. 1000-4.

33. Nakayasu, E.S., et al., Ancient Regulatory Role of Lysine Acetylation in Central Metabolism. MBio, 2017. 8(6). 
34. Barjaktarovic, Z., et al., Hyperacetylation of Cardiac Mitochondrial Proteins Is Associated with Metabolic Impairment and Sirtuin Downregulation after Chronic Total Body Irradiation of ApoE (-/-) Mice. Int J Mol Sci, 2019. 20(20).

35. Santo-Domingo J, Dayon L, Wiederkehr A. Protein Lysine Acetylation: Grease or Sand in the Gears of $\beta$-Cell Mitochondria?. J Mol Biol. 2020. 432(5):p.1446-1460.

36. Chen, L.N., et al., The Brain NO Levels and NOS Activities Ascended in the Early and Middle Stages and Descended in the Terminal Stage in Scrapie-Infected Animal Models. Mol Neurobiol, 2017. 54(3): p. $1786-1796$.

37. Shi, Q., et al., Mouse-adapted scrapie strains 139A and ME7 overcome species barrier to induce experimental scrapie in hamsters and changed their pathogenic features. Virol J, 2012. 9: p. 63.

38. Chen, C., et al., Remarkable increases of alpha1-antichymotrypsin in brain tissues of rodents during prion infection. Prion, 2017. 11(5): p. 338-351.

\section{Figures}

A
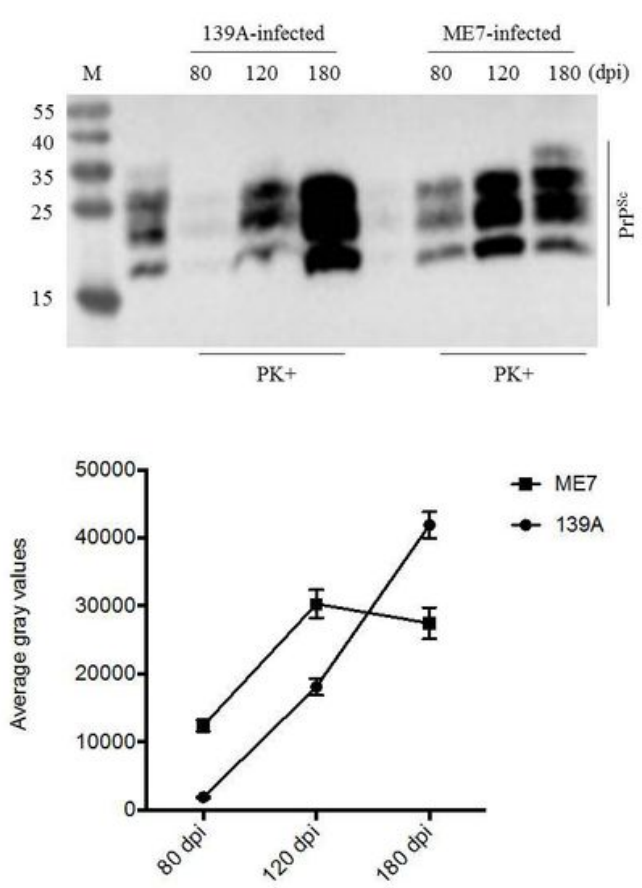

B
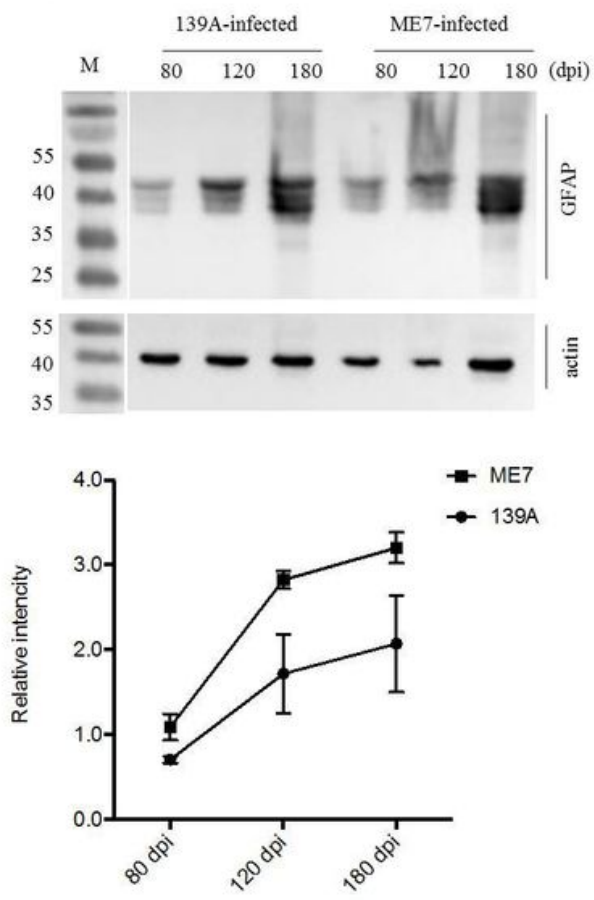

Shi et al, Fig 1
C
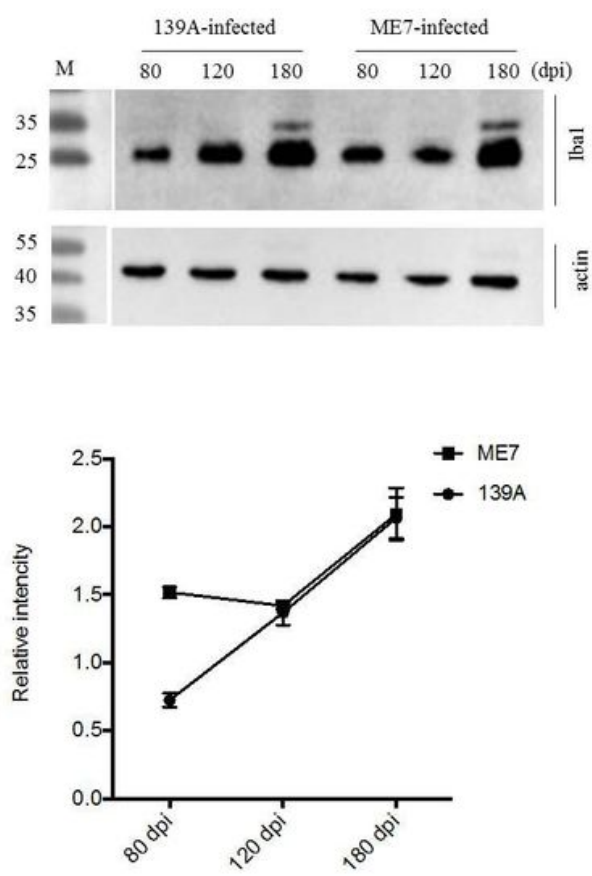

\section{Figure 1}

Western blot evaluations of PrPSc, GFAP and Iba1 in the brain tissues of 139A and ME7 infected mice collected at mid-early, mid-late and terminal stage. The brain homogenates from three individual infected mice collected at different time-points were pooled and used as the representative samples. A. PKdigested Western blot. B. GFAP specific Western blot. C. Iba1 specific Western blot. The quantitative assays of the average intensities of the target bands were showed on the bottom of the individual 
representative images. The relative intensities of GFAP and Ibal were normalized with the data of the individual actin.

A

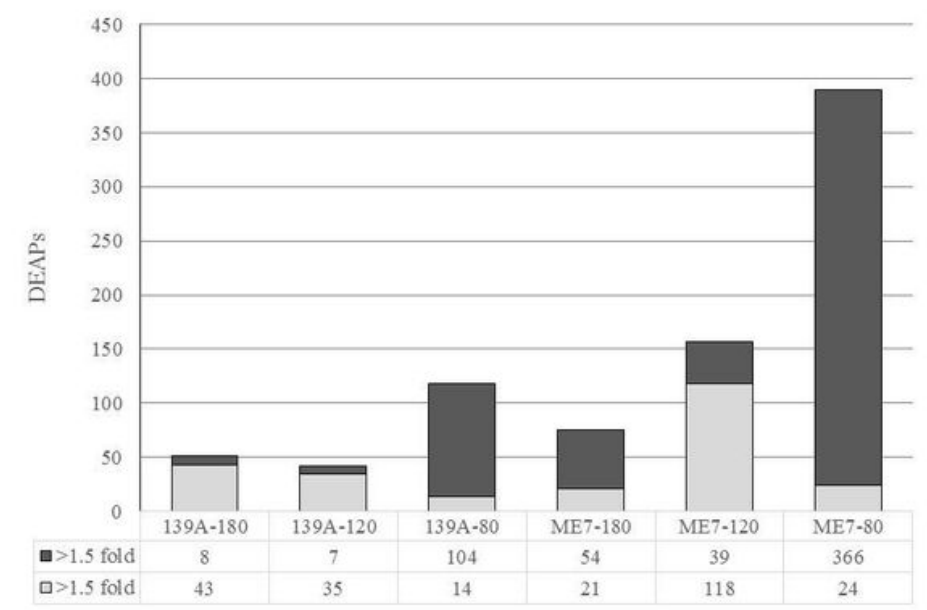

B

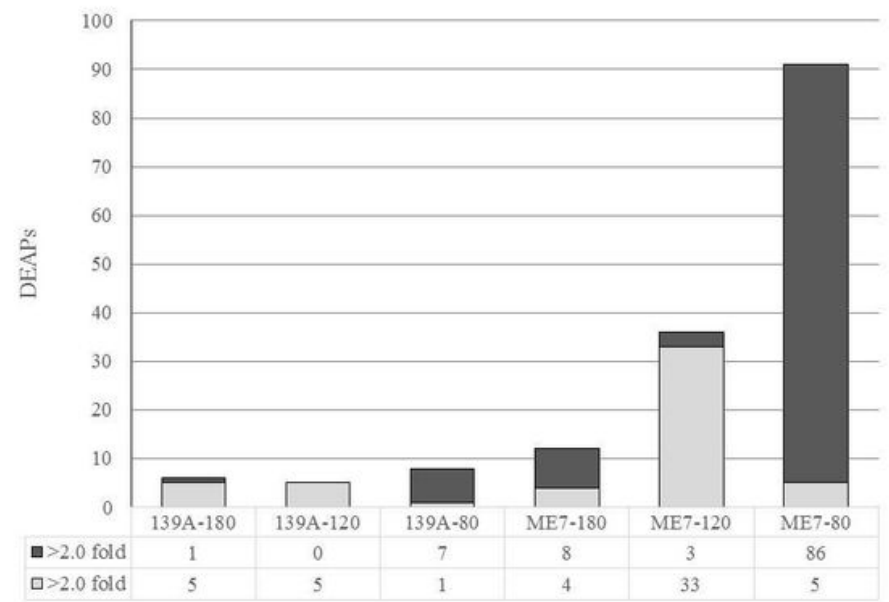

Shi et al, Fig 2

\section{Figure 2}

The numbers of differentially expressed acetylated peptides (DEAPs) in the brains of 139A and ME7 infected mice collected at mid-early, mid-late and terminal stage. A. Based on 1.5-fold change. B. Based on 2.0-fold change. The numbers of increased and decreased DEAPs each sample are shown in the bottom. 
$\mathbf{A}$

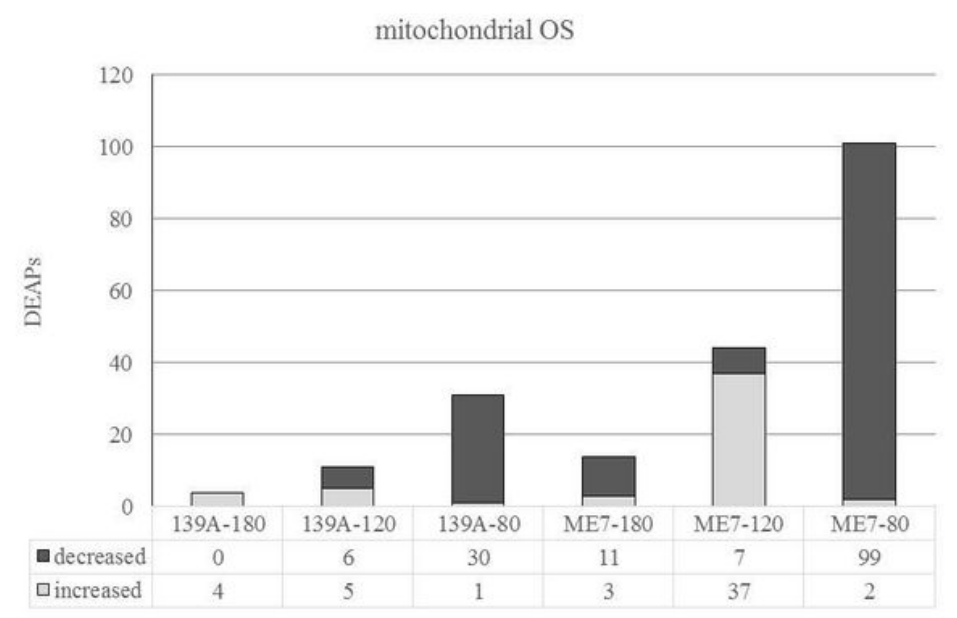

B

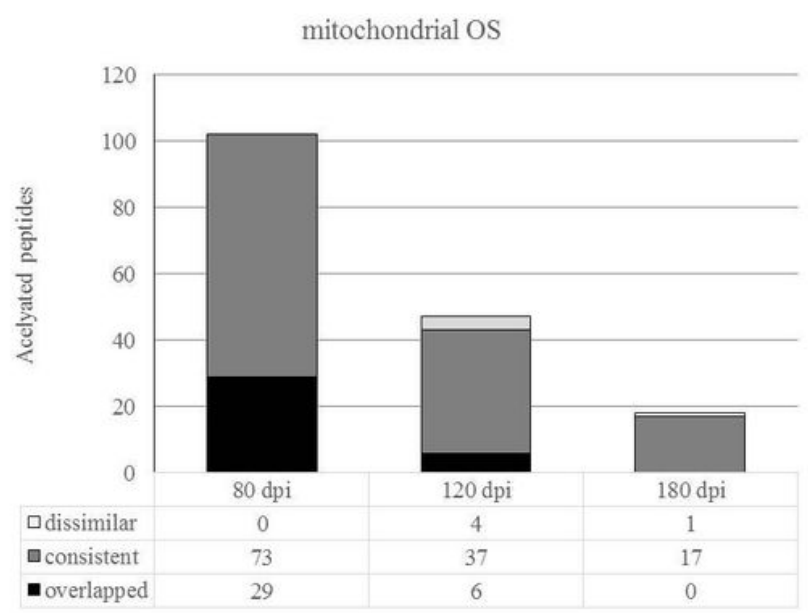

Shi et al, Fig 3

\section{Figure 3}

The mitochondrial associated DEAPs in the brains of 139A and ME7 infected mice collected at mid-early, mid-late and terminal stage. A. The numbers of DEAPs based on 1.5 -fold change. The numbers of increased and decreased DEAPs each sample are shown in the bottom. B. The changing trends of DEAPs. The numbers of overlapped, similar and dissimilar DEAPs each sample are shown in the bottom. 
A

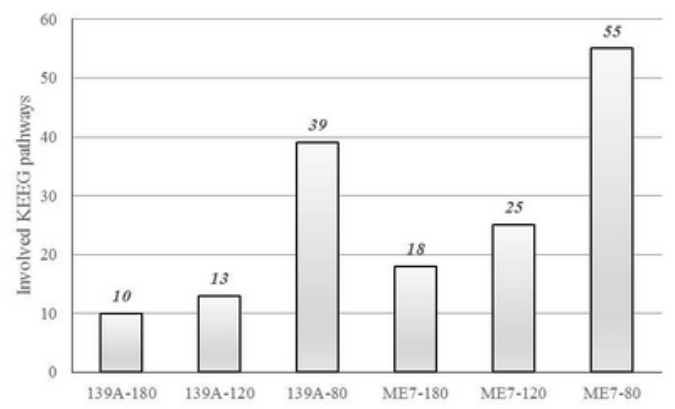

B

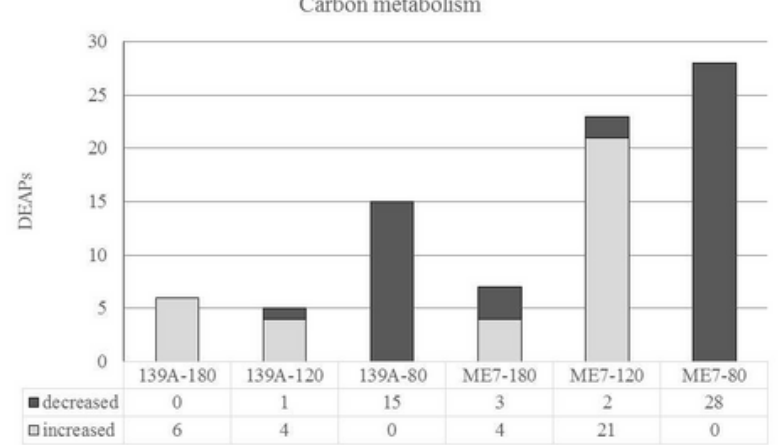

D

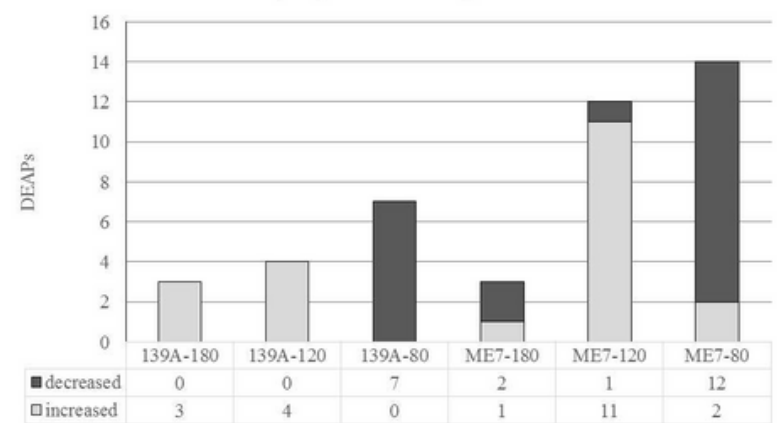

F

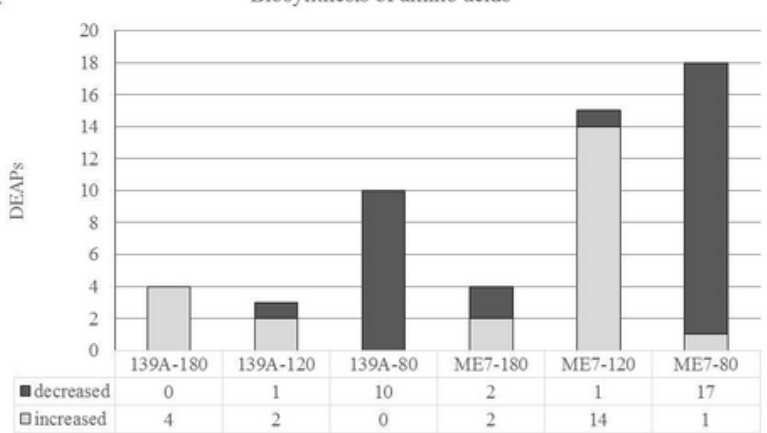

C
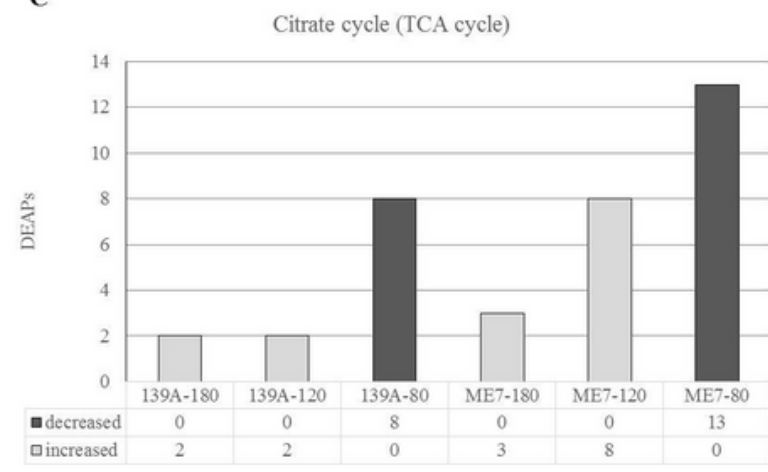

Shi et al, Fig 4

E

Pyruvate metabolism

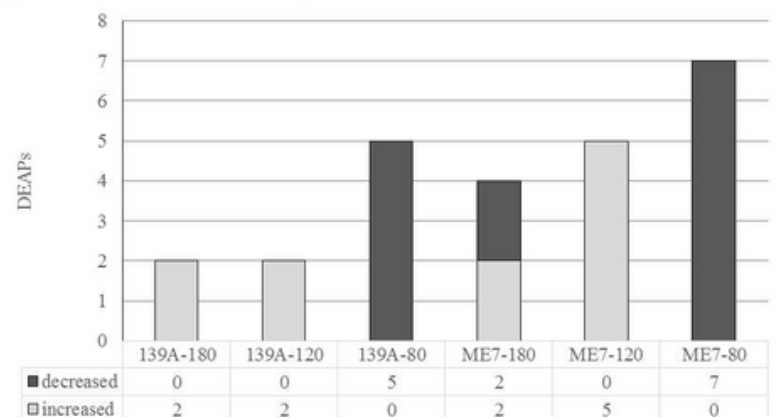

G

Metabolic pathways

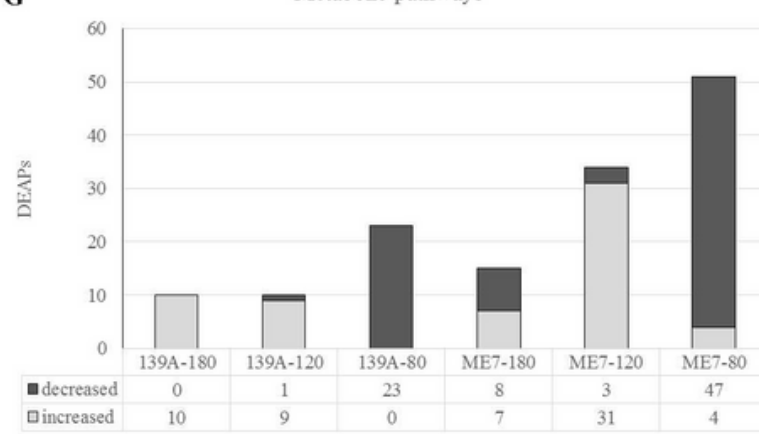

Shi et al, Fig 4

\section{Figure 4}

The numbers of the involved KEEG pathways and the numbers of DEAPs in six commonly-detected pathways in the brains of 139A and ME7 infected mice collected at mid-early, mid-late and terminal stage. A. The KEEG pathways. The numbers of the involved pathways each sample are indicated at top. B. Carbon metabolism. C. TCA cycle. D. Glycolysis / Gluconeogenesis. E. Pyruvate metabolism. F. 
Biosynthesis of amino acids. G. Metabolic pathways. The numbers of increased and decreased DEAPs each sample are shown in the bottom.

A

139A-infected mice

80 dpi

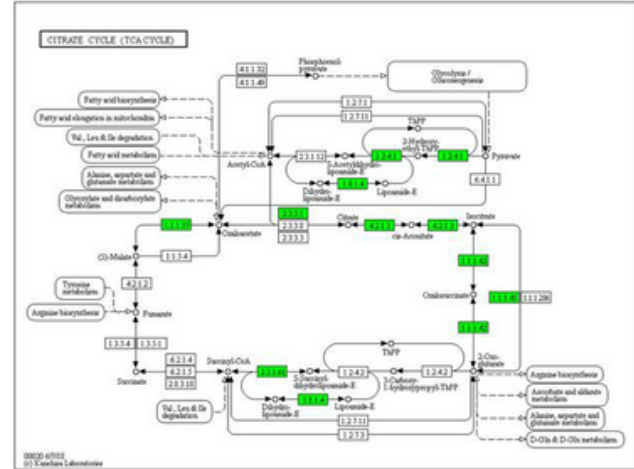

120 dpi

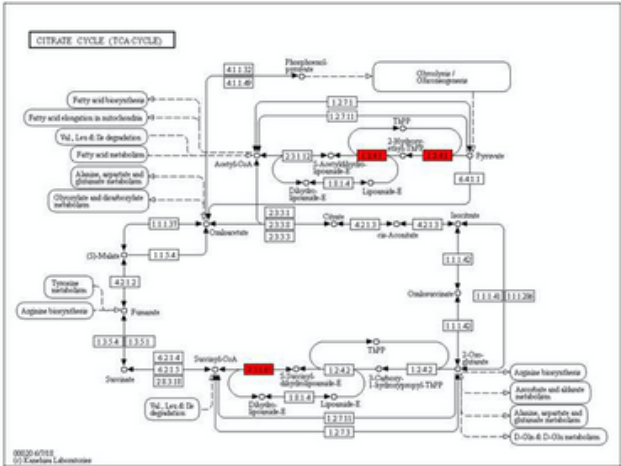

180 dpi

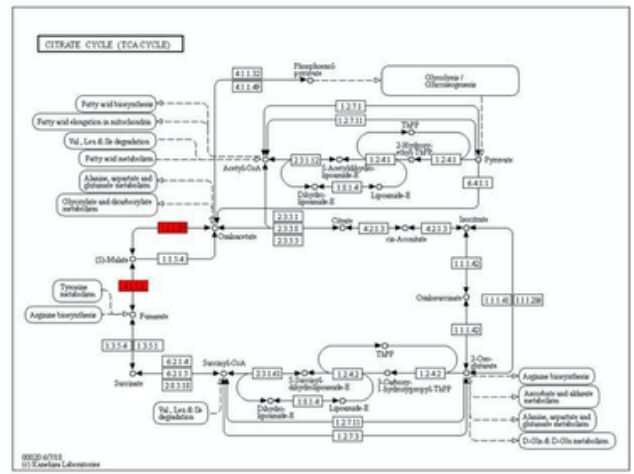

Shi et al, Fig 5

B

ME7-infected mice

80 dpi

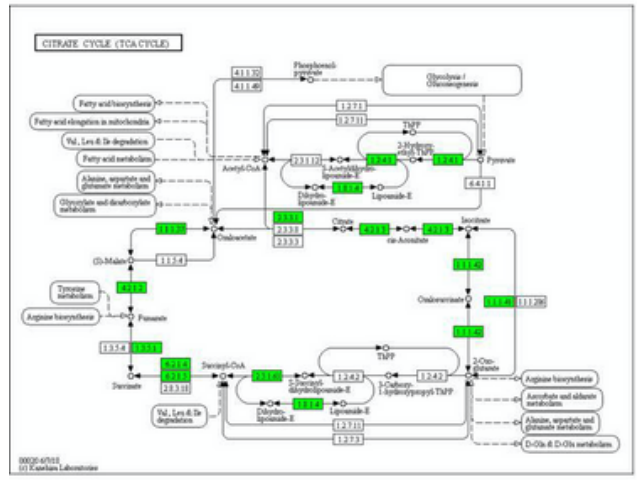

$120 \mathrm{dpi}$

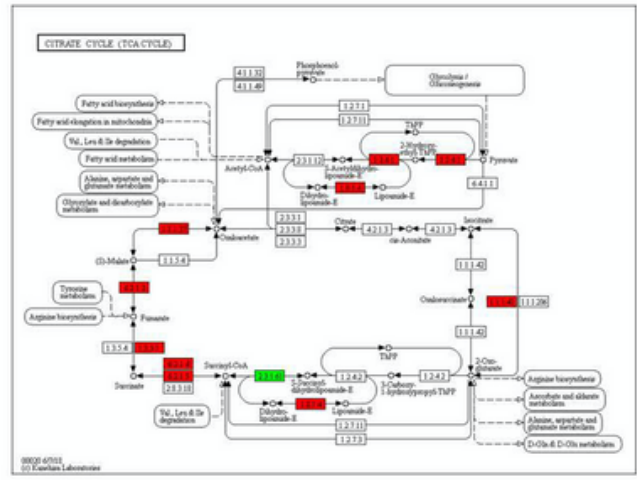

180 dpi

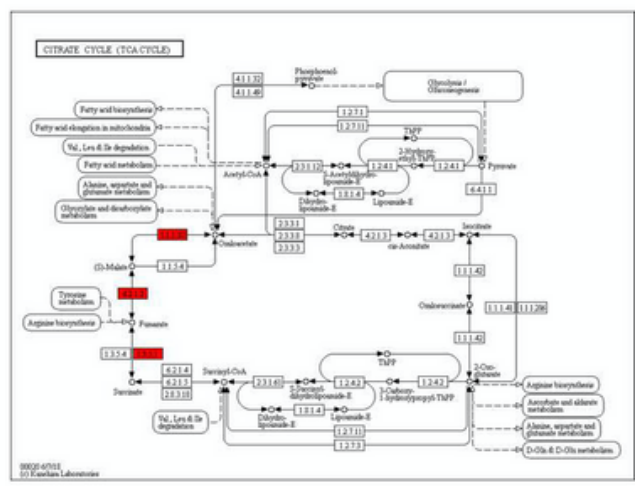

Shi et al, Fig 5

\section{Figure 5}

Analysis of the up- or down-regulated acetylated proteins (enzymes) and catalytic steps in the pathway of TCA cycle in the brains of 139A and ME7 infected mice collected at mid-early, mid-late and terminal 
stage. A. 139A infected mice B. ME7 infected mice. Down-regulated acetylated enzymes are marked in green and up-regulated ones are marked in red.

\section{Supplementary Files}

This is a list of supplementary files associated with this preprint. Click to download.

- Table.pdf

- supplementarymaterials.doc 